\title{
Moisture and Temperature Effects on Interface Mechanical Properties for External Bonding
}

\author{
Ueda Tamon, Justin Shrestha, and Khuram Rashid \\ Division of Engineering and Policy for Sustainable Environment, Faculty of Engineering, \\ Hokkaido University, Japan \\ Zhang Dawei \\ College of Civil Engineering and Architecture, Faculty of Engineering, Zhejiang University, China
}

\begin{abstract}
In order to develop rational guidelines for strengthening by external bonding, it is necessary to clarify longterm performance of interfacial bonding property. In this paper, moisture effects on bonding properties at FRP-concrete interface and temperature/moisture effects on bonding properties at $\mathrm{PCM}$-concrete interface are presented. Shear bond strength of FRP-concrete interface is affected by moisture because resin-concrete adhesion strength is affected by moisture. Among tested CFRP external bonding systems, wet-layup CFRP systems all show the strength reduction, while prefabricated CFRP systems all show the strength increase after immersion. The bond stress-slip relationship and interfacial fracture energy also change, which can explain the change in shear bond strength. The reduction in shear bond strength does not show clear dependency on resin strength/stiffness reduction. The observed big variation in shear bond strengths reported in past studies can be explained by difference in interface roughness, since chemical bond is affected by moisture but mechanical bond is not. Tension/shear bond strength of PCM-concrete interface is affected significantly by temperature but less by moisture. The tensile/shear bond strength is less than tensile/shear strength of constituent materials (PCM and concrete). The reduction in tension/shear bond strength depends on the reduction in tensile/shear strengths of $\mathrm{PCM}$ and concrete and then estimated by proposed equation which is a function of PCM and concrete strength. The ultimate loads of beams strengthened by PCM overlay can be predicted by the proposed model in which the proposed tensile/shear bond strength is applied. Under high temperature, the failure mode can be changed from flexure/shear failure to debonding failure due to the reduction in shear bond strength.
\end{abstract}

\section{INTRODUCTION}

We have been constructing many infrastructures including buildings all over the world. In many countries, damages of those structures due to environmental actions and natural/manmade disasters are a big social problem. Many existing structures do not satisfy the present requirements by design standards, so that safety and serviceability of those structures is a big concern to the society.

Sustainable solution to tackle with non-conforming structures with and without damages is repair/ strengthening of structures. Repair/strengthening can extend life of existing structures with less resources. Typical method for repair/strengthening is external bonding and overlaying. Debonding at interface between reinforcing material and substrate concrete is a failure mode, which is unique and cannot be seen in structures without external bonding and overlaying. Thus, there have been many studies on debonding, which disclose three debonding failure modes; (1) intermediate crack debonding, (2) concrete cover separation, and (3) plate end peeling. Past studies present debonding strength prediction methods for these failure modes. However, deterioration of interface debonding strength due to environmental actions has not fully understood yet. For example, past studies on effects of moisture on debonding strength of interface between fiber reinforced polymer (FRP) and concrete indicate quite different findings on strength reduction. On the other hand, there is practically no past study on effects of temperature on debonding strength of interface between polymer cementitious mortar (PCM) and concrete.

Our research group has been investigating the effects of moisture and temperature on interface bonding property. In this paper, the following two are briefly introduced; (1) moisture effects on bonding properties at interface between FRP and concrete and (2) temperature and moisture effects on bonding properties at interface between PCM and concrete. 


\section{MOISTURE EFFECTS ON BONDING PROPERTIES AT INTERFACE BETWEEN FRP AND CONCRETE}

In the past studies on moisture effects on FRPconcrete interface, mostly carbon FRP was used with different systems provided by different companies. In this study, therefore, six types of CFRP external bonding systems, which are internationally well accepted three Japanese, two European, and one North American systems, were tested. The tested specimens for bond property as shown in Figure 1 and dog-bone adhesive/primer resin specimens were fully immersed in water until testing. The immersion period was 18 months. The resin specimens absorbed moisture continuously for 18 months (see Figure 2) and showed reduction in tensile strength (see Figure 3 ). However, the relationship between absorbed moisture amount and reduction in tensile strength depends on the type of resin (see Figure 3). Some resins show the reduction, while some show the increase. The shear bond strength also depends on the types of externally bonded CFRP systems. Wet-layup types (four types) show reduction in shear strength, while dry-layup types (preimpregnated types) (two types) shows some increase in shear strength. The extent of reduction and increase depends on the type of CFRP systems (see Figure 4). It was considered that resin stiffness softened due to moisture may explain the enhancement of shear strength. In the case where the shear strength reduction was observed, the failure

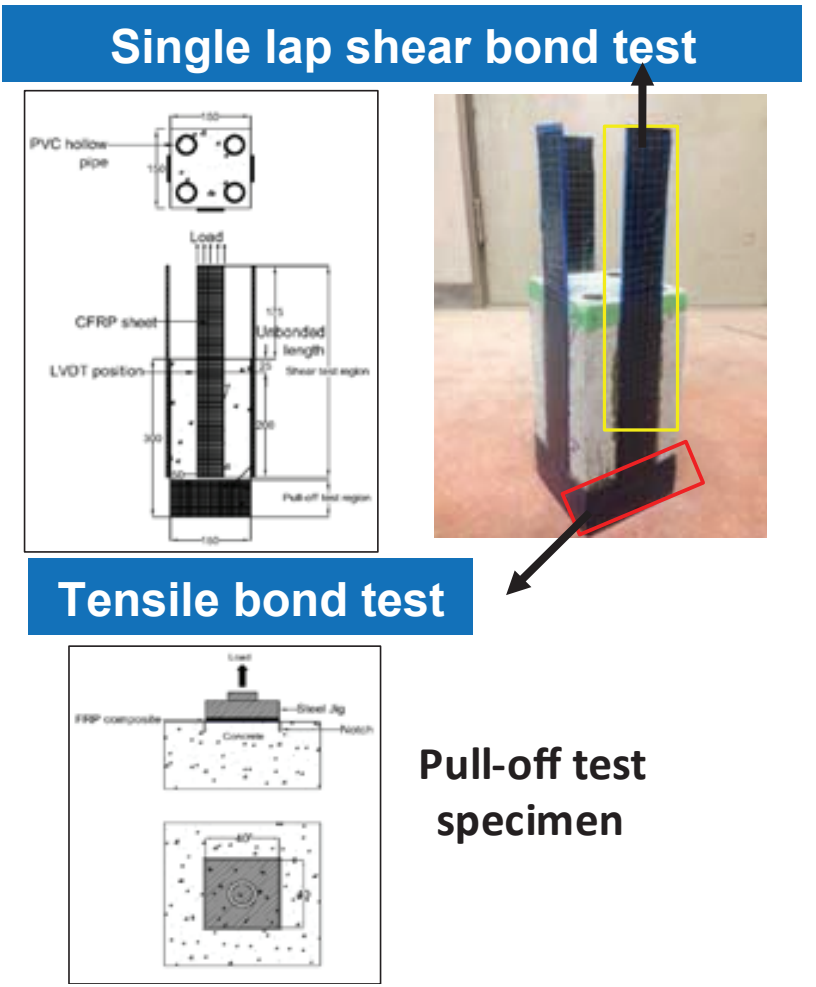

mode was changed to resin-concrete adhesion failure. Tensile bond strength was reduced in all six CFRP types.

In order to investigate effects of interface pretreatment (surface roughness) on bond strength, the shear bond strength of a wet-layup type was examined after 12 months of immersion. Specimens with lower roughness treated by disk-sander show greater reduction $(30 \%)$ in bond strength, while specimens with higher roughness by sand-blasting show less reduction $(7 \%)$ (see Figure 5$)$. In the former case the failure mode was changed from mixed mode (concrete cohesion with adhesion) to adhesion mode. These facts indicate that at the interface mechanical bond does not deteriorate but chemical bond does.

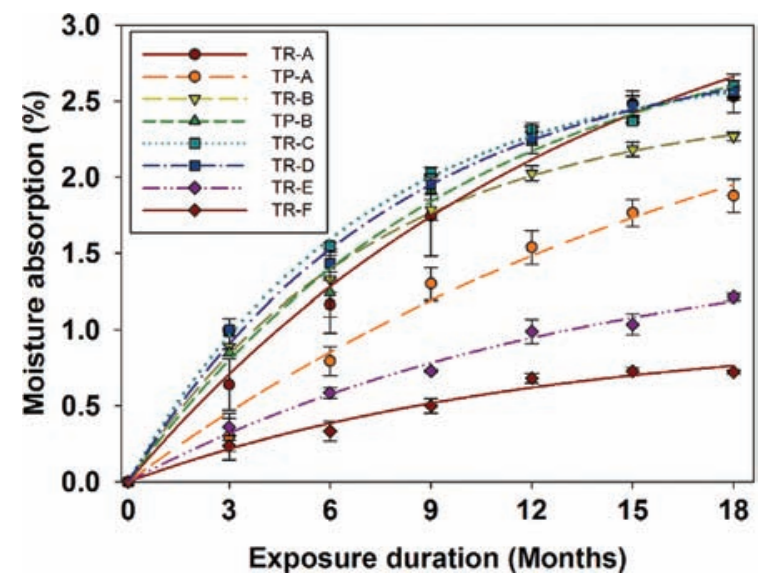

Figure 2. Moisture absorption by resin.

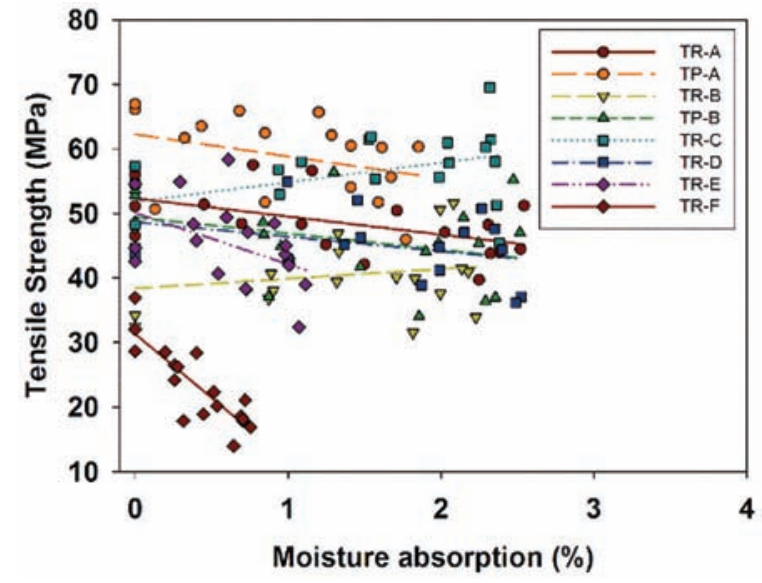

Figure 3. Moisture absorption and tensile strength of resin.

The deterioration of bond due to moisture can be described by bond stress-slip relationship $(\tau-s)$, which can be obtained from FRP strain-slip relationship $(\varepsilon-s)$ as shown below (Dai, Ueda, \& Sato, 2005);

$$
\varepsilon=f(s)=A\left(1-e^{(-B s)}\right)
$$

Figure 1. Specimens for bond property. 

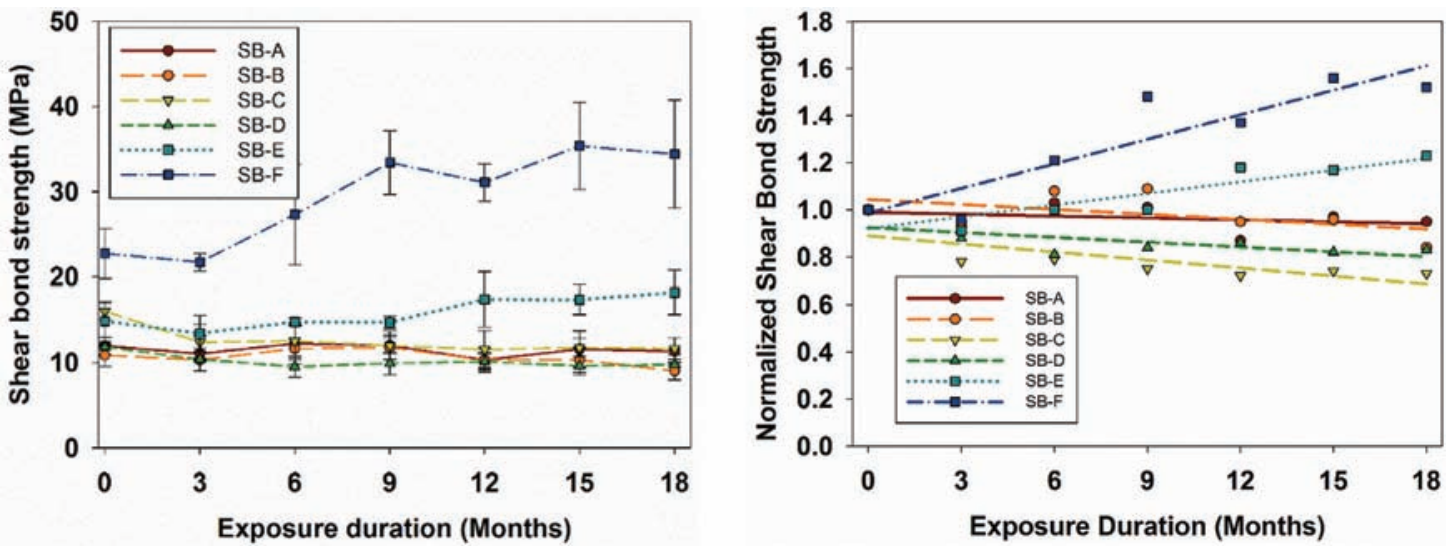

Figure 4. Shear bond strength after immersion.

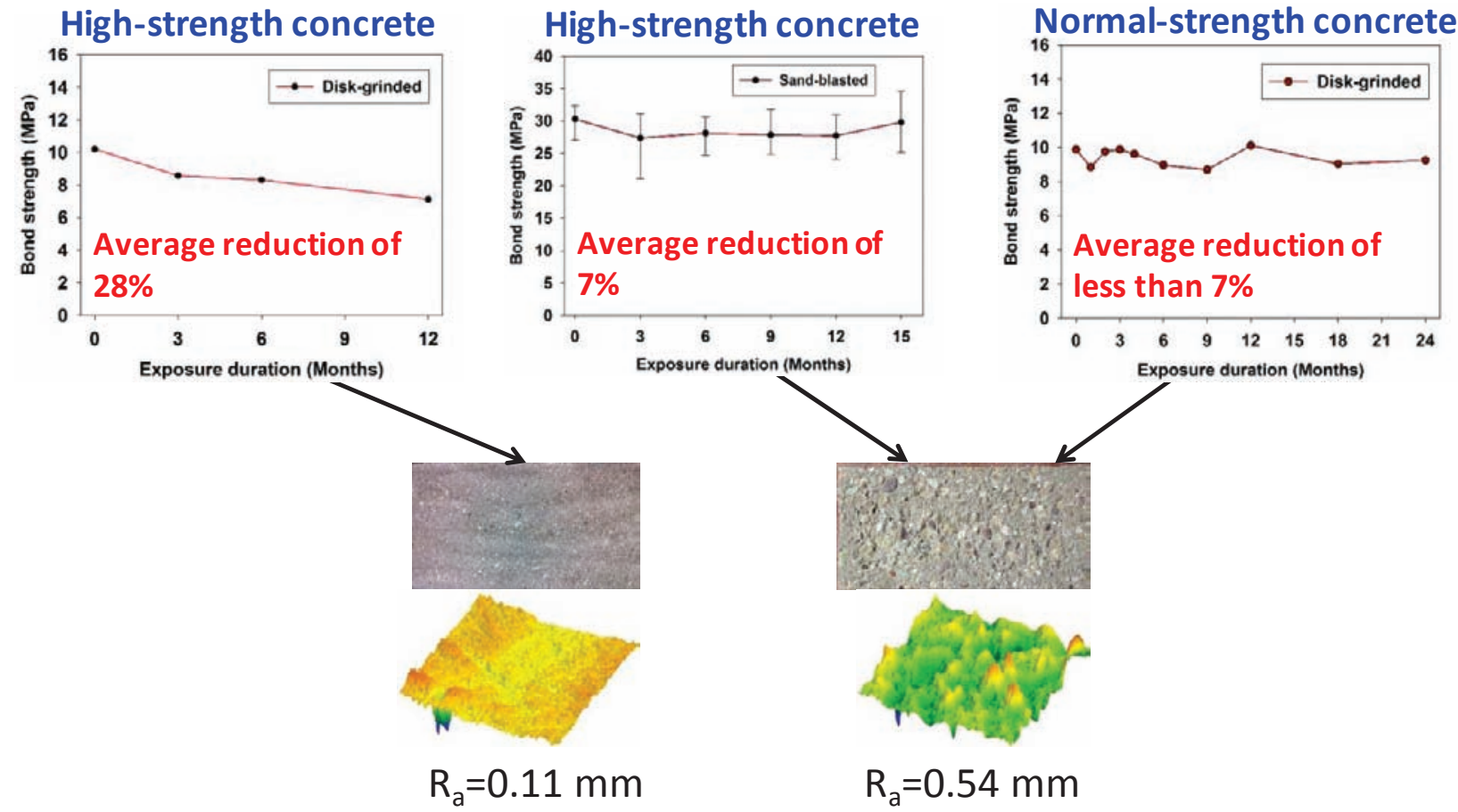

Figure 5. Effects of surface roughness on bond strength reduction due to moisture.

$$
\tau=A^{2} B E_{f} t_{f} e^{(-B s)}\left(1-e^{(-B s)}\right)
$$

From Eq. (2), the interfacial fracture energy can be obtained as below;

$$
G_{f}=\frac{1}{2} A^{2} E_{f} t_{f}
$$

The effects of moisture immersion period $(t)$ for the wet-layup types can be represented by the ductility parameter $(B)$ and fracture energy $(G)$ as below;

$$
\begin{aligned}
& \left(B_{t}^{E n v .}\right)_{w}=B_{0}\left(1.05-0.05 e^{-0.40 t}\right) \\
& \left(G_{f t}^{E n v .}\right)_{w}=G_{f}\left(0.8+0.2 e^{(-0.38 t)}\right) 0
\end{aligned}
$$

where $B_{0}$ and $G_{f 0}$ are the values for the case without water immersion. The decrease in fracture energy for the wet-layup types can be seen in Figure 6, while the variation in bond stress-slip relationship is shown in Figure 7. The effects of moisture exposure appear for the first 6 months and remain rather constant after that.

\section{TEMPERATURE AND MOISTURE EFFECTS ON BONDING PROPERTIES AT INTERFACE BETWEEN PCM AND CONCRETE}

Effects of temperature on PCM are generally known, however, precise information of each PCM available in actual market is quite limited. Almost no information 


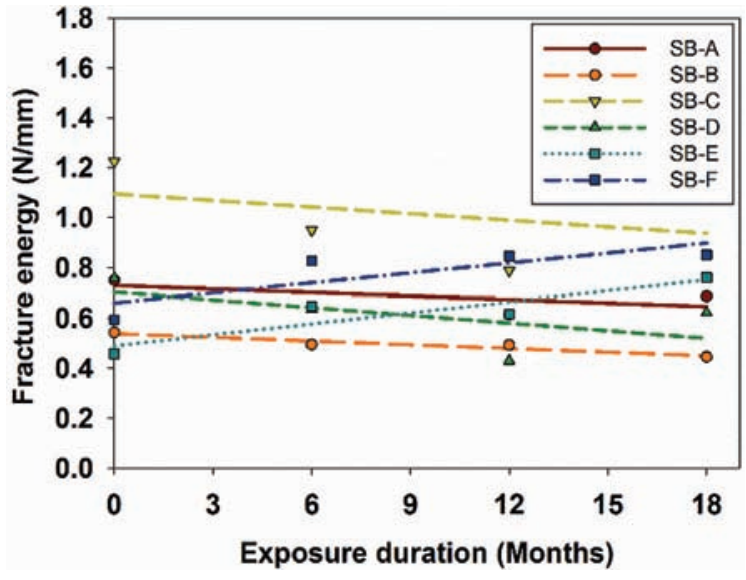

Figure 6. Relationship between interfacial fracture energy and moisture exposure duration.

is available for temperature and moisture effects on interface property between PCM and concrete. Therefore, experimental investigation on PCM and PCM-concrete interface properties under temperature and moisture exposure conditions using four different PCM commercially available in Japan and China was conducted. The results show that general tendency on effects of temperature and moisture on bonding properties is the same among those different PCM cases as shown below.

In order to see tendency of temperature effects on interface property, tension bond tests were conducted using $\mathrm{PCM}$-concrete composite prism specimens with the first type of PCM (see Figure 8). Test results are shown in Figure 9. The exposure to $60^{\circ} \mathrm{C}$ in air reduces tensile bond strength by $30 \%$ after 1 day but does not reduce further till 30 days. The tensile bond strength measured at $20^{\circ} \mathrm{C}$ after $60^{\circ} \mathrm{C}$ exposure is increased from the strength tested as $60^{\circ} \mathrm{C}$ but not fully recovered. Some strength increase takes place after 30 days due to further hydration of PCM. Adopted temperature cyclic conditions were TC1 which consists of $60^{\circ} \mathrm{C}$ in air for $12 \mathrm{~h}$ and $30^{\circ} \mathrm{C}$ in air for $12 \mathrm{~h}$ and $\mathrm{TC} 2$, which consists of $24 \mathrm{~h}$ of $60^{\circ} \mathrm{C}$ in air, $24 \mathrm{~h}$ of $20^{\circ} \mathrm{C}$ in water, $24 \mathrm{~h}$ of $0^{\circ} \mathrm{C}$ in air, and $24 \mathrm{~h}$ of $25^{\circ} \mathrm{C}$ in air. TC1 represents a climatic change for 1 day, while TC2 represents a climatic change for 1 year. Thirty and 10 cycles were applied for TC1 and TC2, respectively. The reduction in tensile bond strength is more than that under constant $60^{\circ} \mathrm{C}$ exposure.

The second and third types of PCM were used to examine combined effects of temperature and moisture on PCM-concrete interface strength. Figure 10 shows experiment outline. Tensile bond strength at PCM-concrete interface is less than tensile strength of PCM and concrete under any testing condition (see Figure 11). However, bond strength reduction in $\%$ due to temperature increase from 20 to $60^{\circ} \mathrm{C}$ is smaller than that of PCM. The effects of temperature on tensile bond strength are almost same between wet and dry conditions. Effects of moisture on tensile bond strength are hardly seen under wet/dry cycles and continuous immersion at $20^{\circ} \mathrm{C}$.

The interfacial bond tests of fourth type of PCM, which was used for investigation of structural performances of beams strengthened by PCM overlaying, was conducted (see Figure 12). There were two cases of
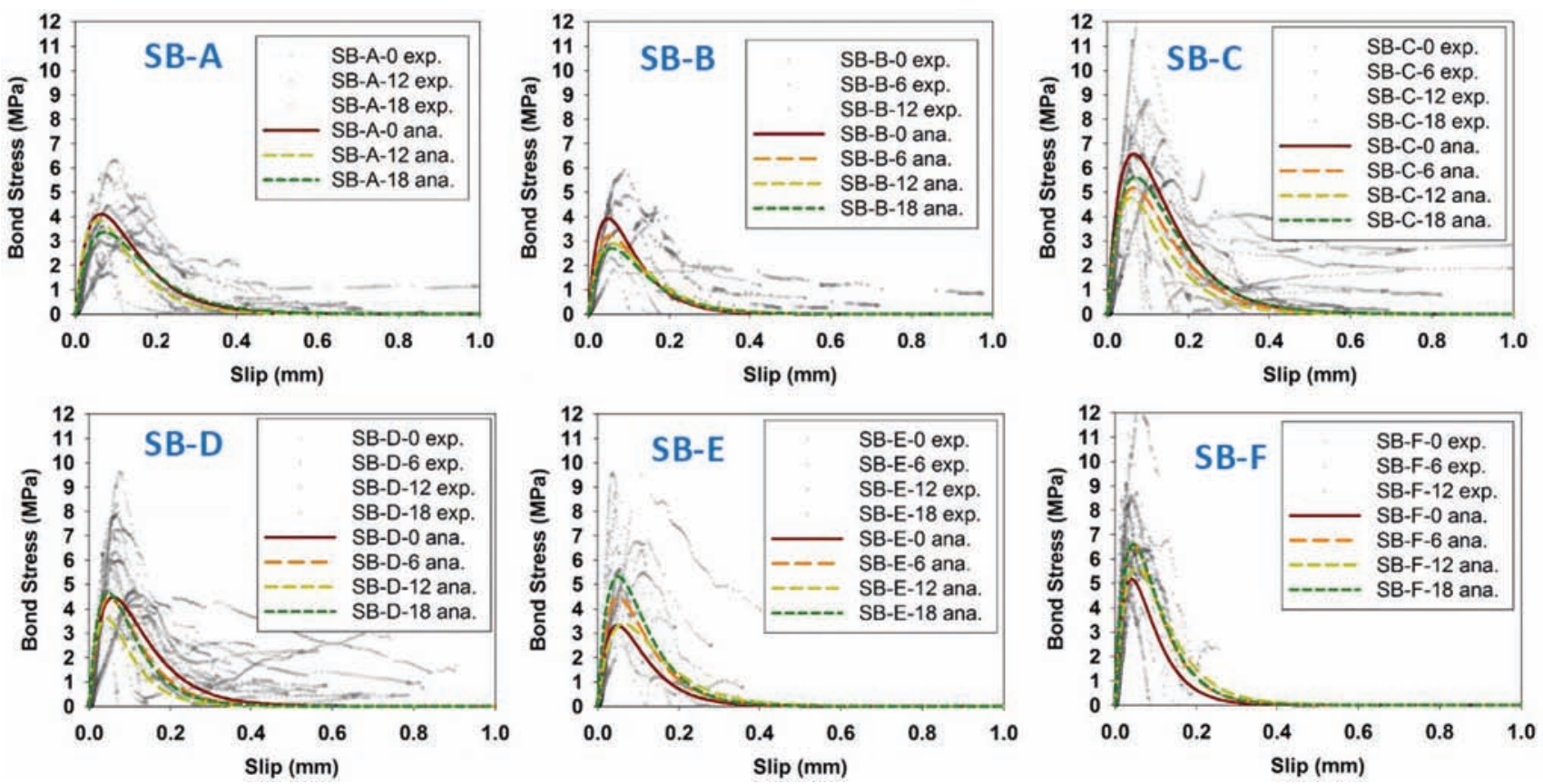

Figure 7. Effects of moisture exposure and comparison of predicted bond-slip curves with experimental curves for wet-layup types. 


\section{Specimen Preparation}
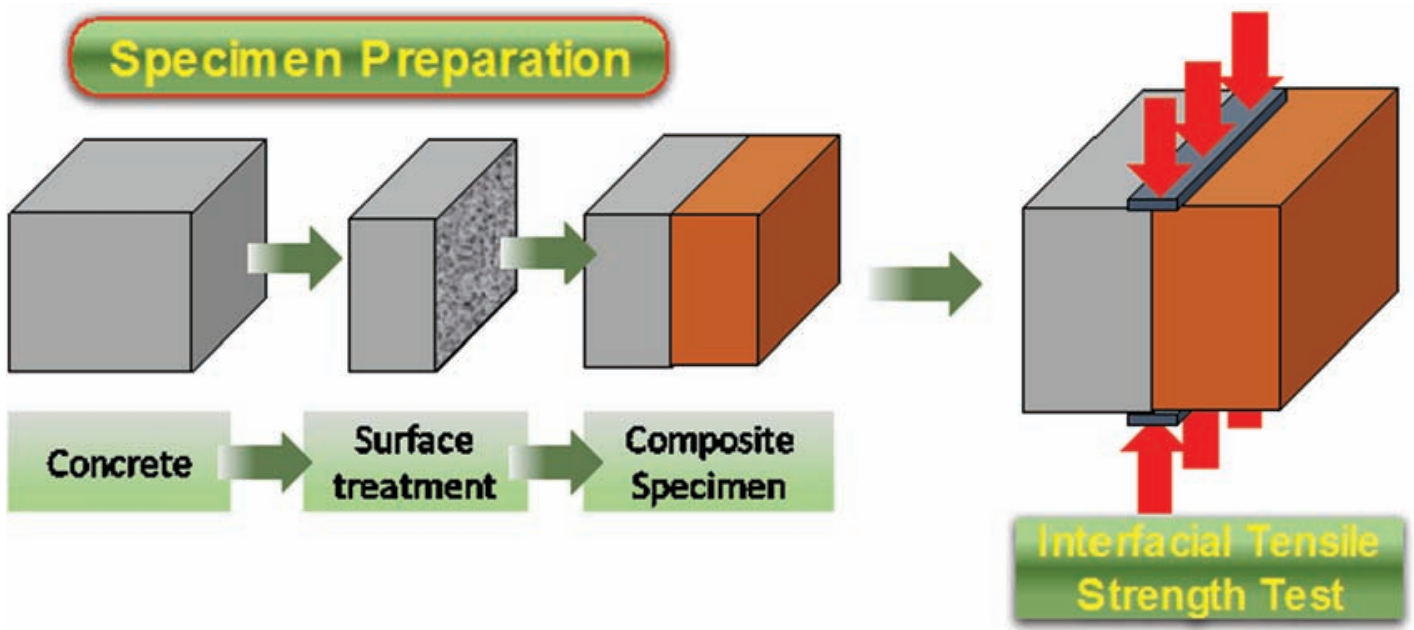

Figure 8. Interfacial tensile strength specimen and loading condition.
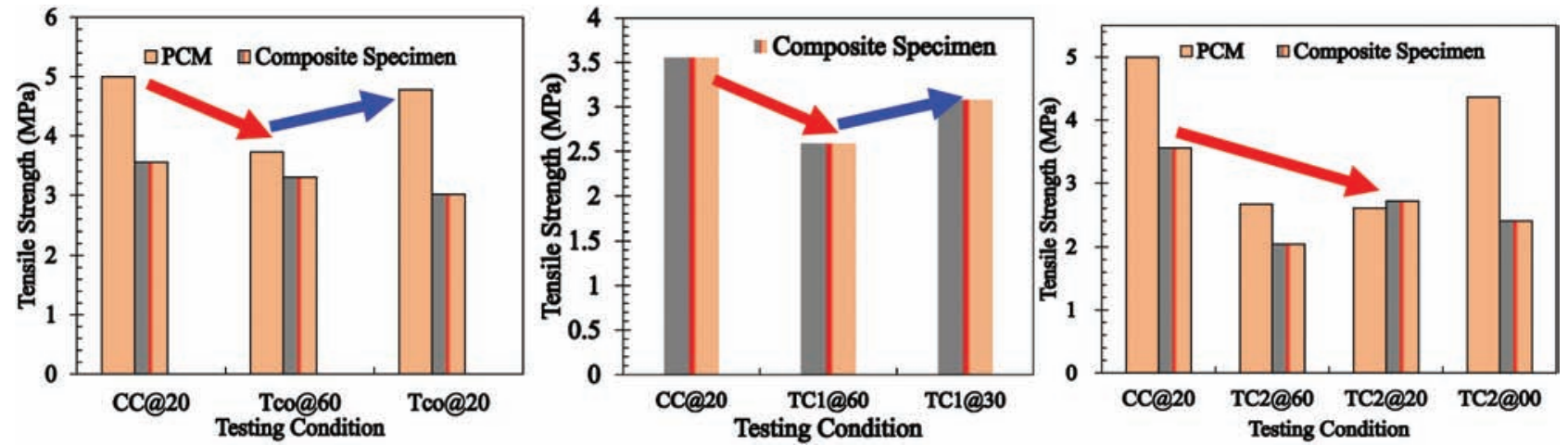

Figure 9. Interfacial and PCM tensile strength under various temperature conditions.

surface treatments with and without primer. Tensile strength of the PCM is larger than the other three types of PCM and interfacial roughness is apparently higher than those for the other PCM-concrete composite specimens. Those facts are considered to be the reason why tensile bond strength is higher than those in the other PCM-concrete composite specimens. Not only tensile bond strength but also shear bond strength decrease with increase in temperature (see Figure 13). Considering the observed relationship between strengths of constituent materials (PCM and concrete) and bond strength at their interface, the following Eqs (6) and (9) can be proposed to estimate tensile and shear bond strength:

$$
\frac{1}{f_{I T S}}=\frac{A_{t}}{f_{t . p c m}}+\frac{B_{t}}{f_{t . c o n c}}
$$

where

$f_{\text {ITS }}: \quad$ Tensile bond strength at concerned temperature (MPa)

$f_{\text {t.pcm }}: \quad$ Tensile strength of $\mathrm{PCM}$ at concerned temperature (MPa)

$$
=1.2 f_{t o . p c m} \exp (-0.0095 T)
$$

$f_{\text {t.conc }}: \quad$ Tensile strength of concrete at concerned temperature (MPa)

$$
=1.07 f_{\text {to, conc }} \exp (-0.004 T)
$$

$f_{\text {to.pcm }}$ : Tensile strength of PCM after 28 days standard curing condition (MPa)

$f_{\text {to.conc }}: \quad$ Tensile strength of concrete after 28 days standard curing condition (MPa)

T: $\quad$ Temperature $\left(20^{\circ} \mathrm{C}<T<60^{\circ} \mathrm{C}\right)$

$A_{t}$ and $B_{t}:$ Experimental coefficients in tension

$$
\frac{1}{\tau_{\text {ISS }}}=\frac{A_{v}}{\tau_{v . \text { pcm }}}+\frac{B_{v}}{\tau_{v \text { vonc }}}
$$

where

$\tau_{I S S}: \quad$ Shear bond strength at concerned temperature (MPa)

$\tau_{v \text {. pcm }}: \quad$ Shear strength of PCM at concerned temperature (MPa) 

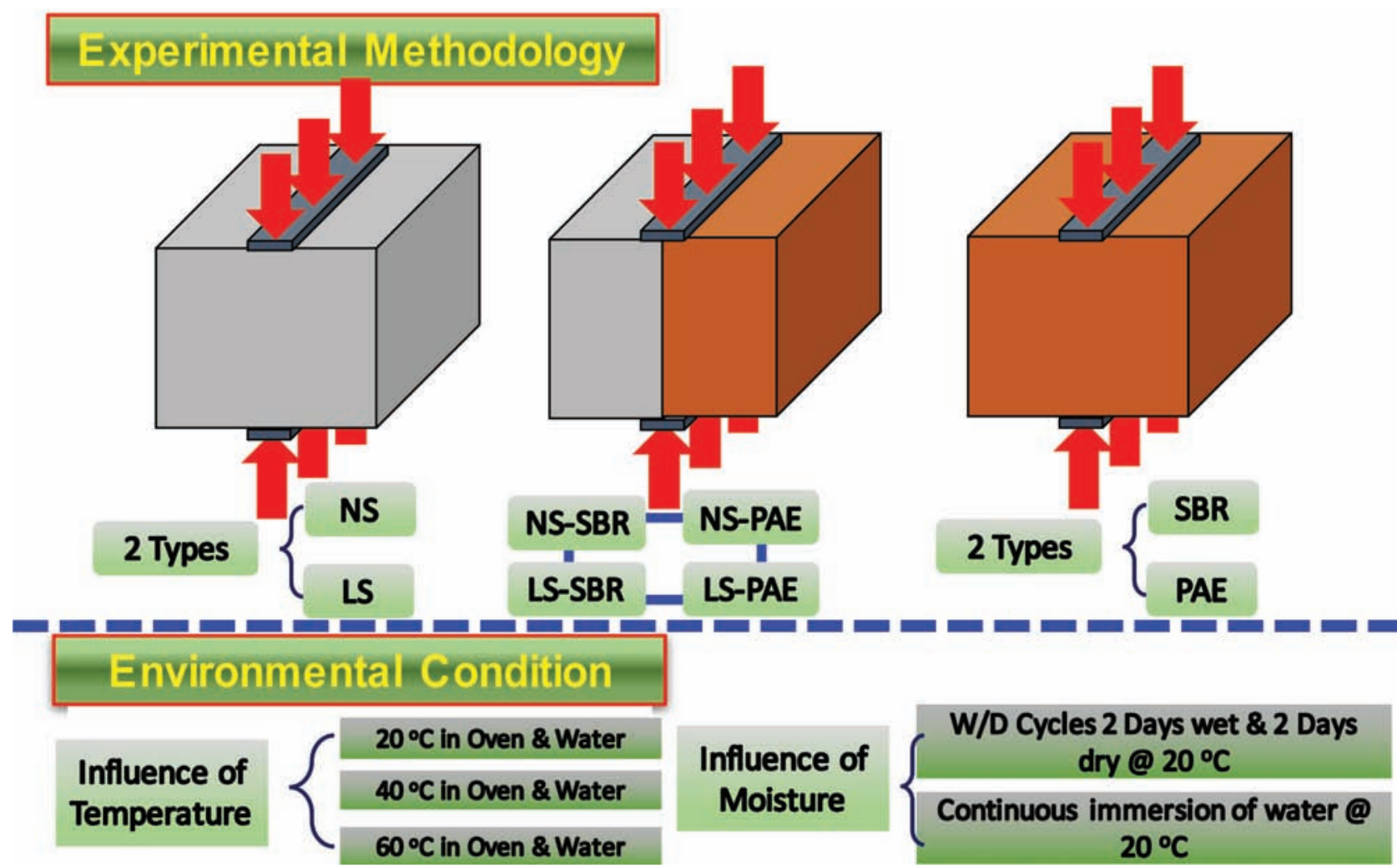

Figure 10. Experimental outline for combined effects of temperature and moisture.
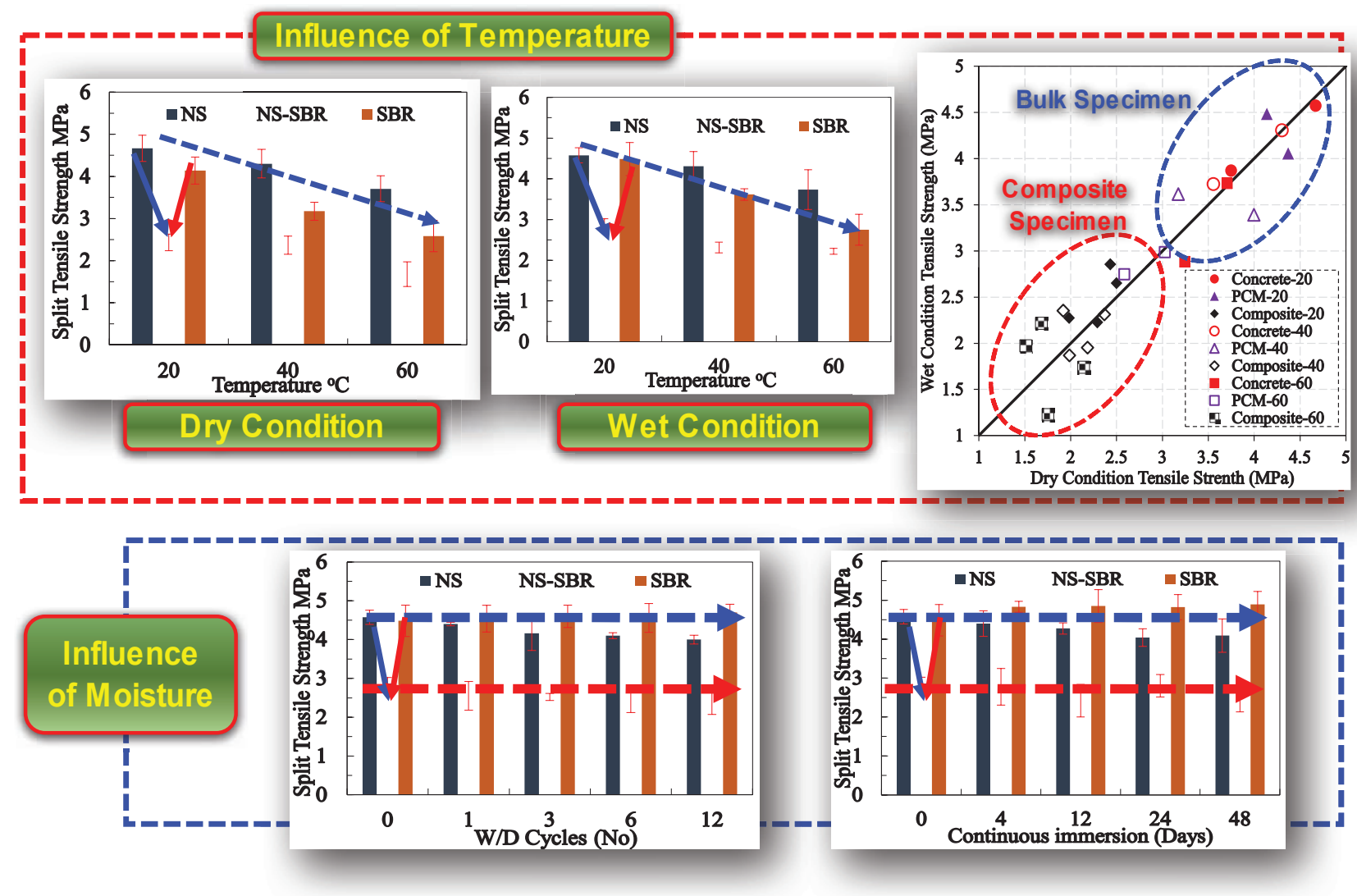

Figure 11. Experimental results under combined effects of temperature and moisture. 


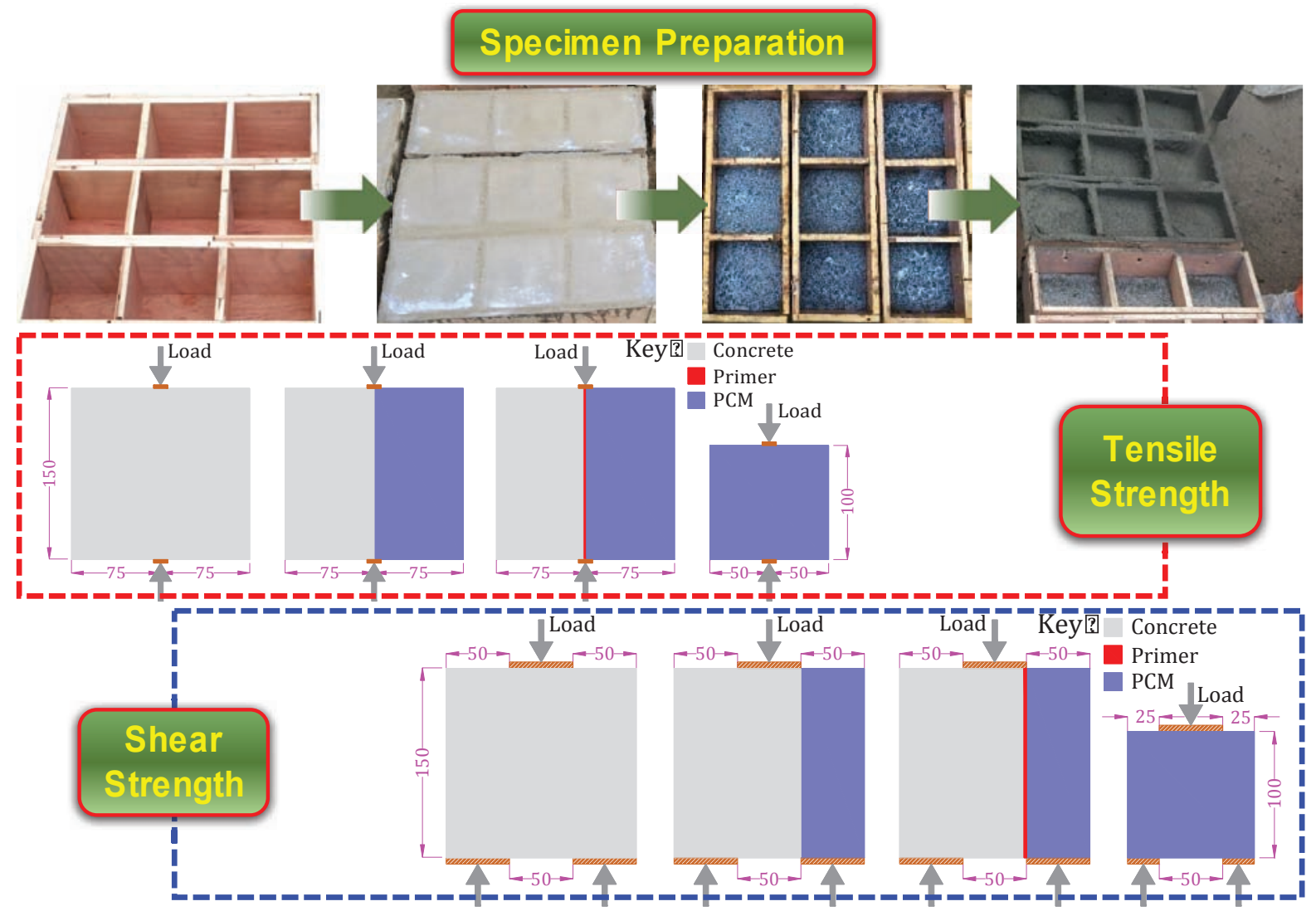

Figure 12. Experimental outline for bond tests of interface in beam strengthened by PCM overlaying.
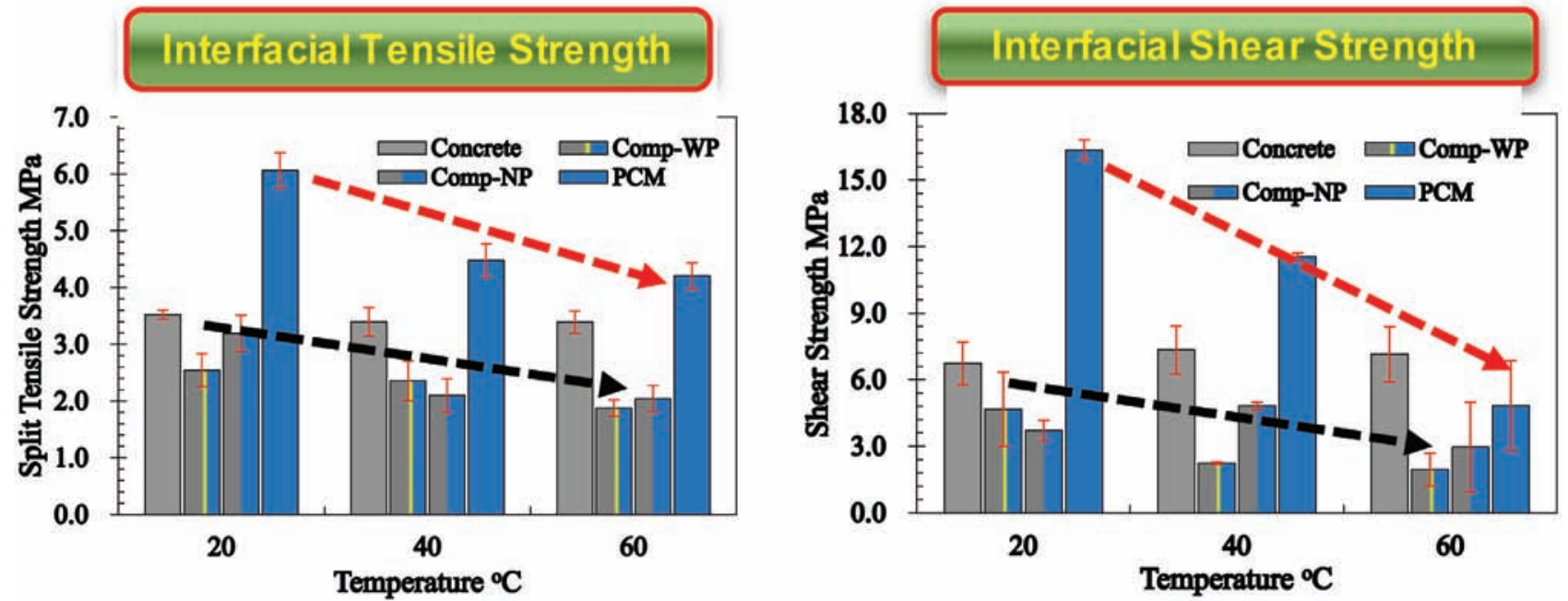

Figure 13. Effects of temperature on both tensile and shear bond strength.

$$
=2 \tau_{\text {vo.pcm }} \exp (-0.03 T)
$$

$\tau_{v . \text { conc }}: \quad$ Shear strength of concrete at concerned temperature $(\mathrm{MPa})$

$$
=0.99 \tau_{\text {vo,conc }} \exp (0.0015 T)
$$

$\tau_{\text {vo.pcm }}: \quad$ Shear strength of PCM after 28 days standard curing condition (MPa) $\tau_{\text {vo.conc }}: \quad$ Shear strength of concrete after 28 days standard curing condition (MPa)

$T: \quad$ Temperature $\left(20^{\circ} \mathrm{C}<T<60^{\circ} \mathrm{C}\right)$

$A_{v} \& B_{v}: \quad$ Experimental coefficients in shear

Figure 14 shows the comparison of predicted bond strength and experimental strength for the second, third, and fourth types of PCM. Good agreement can 

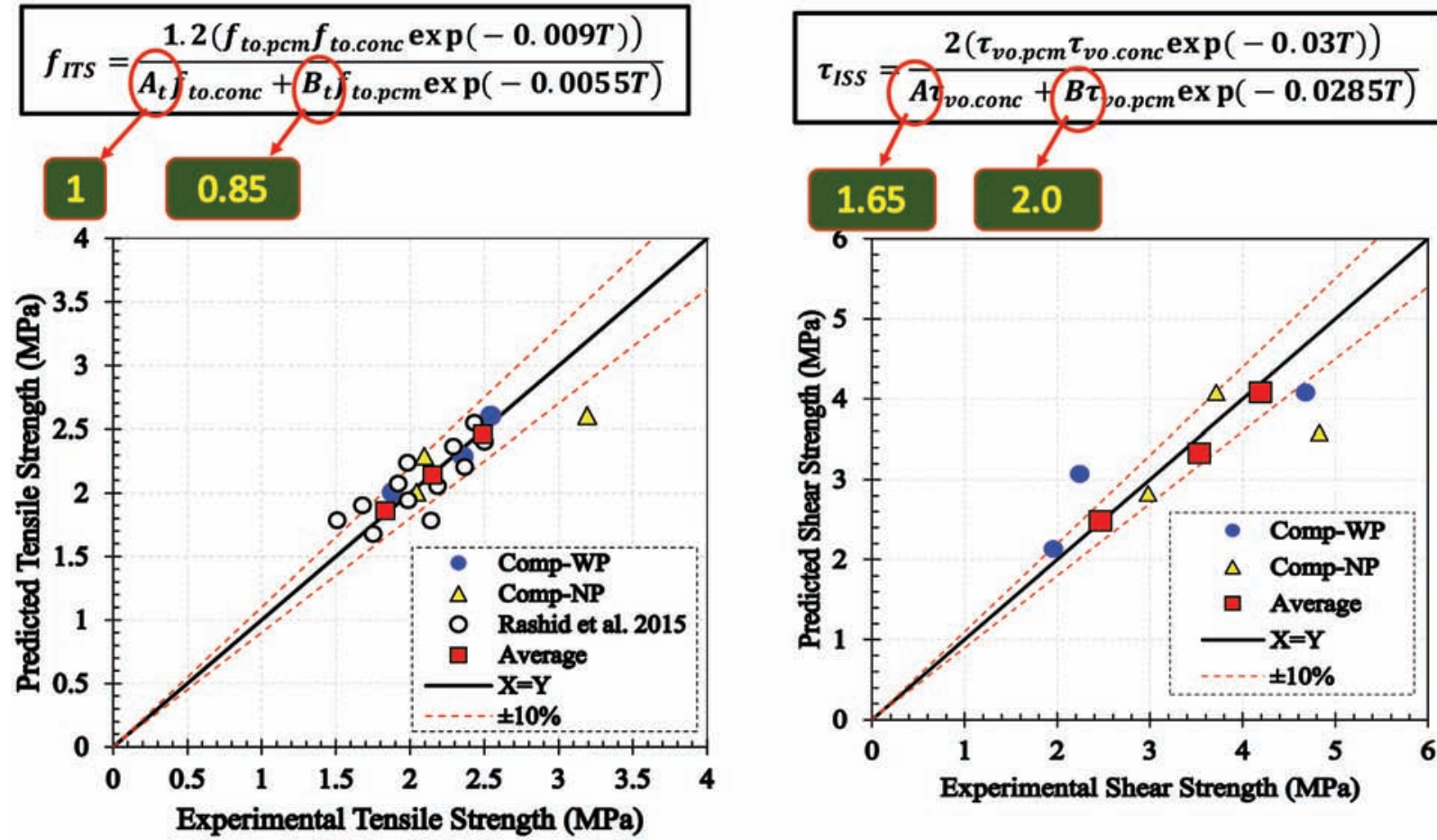

Figure 14. Comparison between predicted and experimental tensile and shear bond strengths.

be seen for both cases with and without primer (-WP and $-\mathrm{NP}$ ).

In order to investigate effects of temperature on debonding strength of beams strengthened in flexure by PCM overlaying, loading tests of 30 beam specimens were conducted with tension reinforcement amount in overlay as a main parameter (see Figure 15). Fifteen specimens were with primer treatment, while the remaining 15 specimens were without primer treatment. Out of 15 specimens, 5 specimens were tested under 20,40 , and $60^{\circ} \mathrm{C}$, respectively. The peak load increases with the increase in tension reinforcement amount in PCM overlay, but decreases with the increase in temperature as shown in Figure 16. Failure mode of the strengthened beams were flexure failure without debonding in the cases with small tension

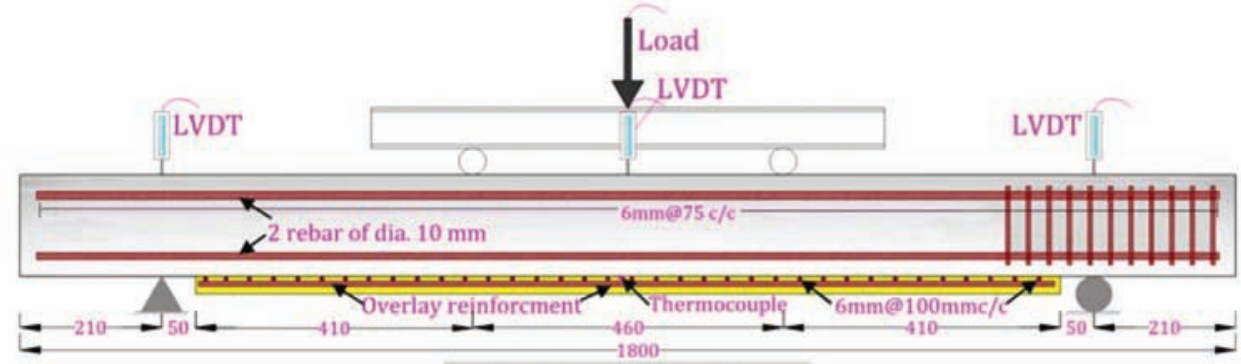

Longitudinal section

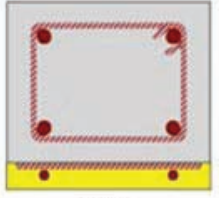

$2 \emptyset 6$

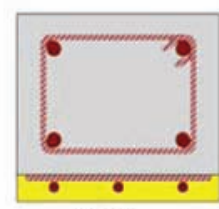

$3 \emptyset 6$

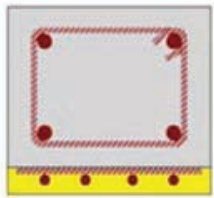

$4 \emptyset 6$

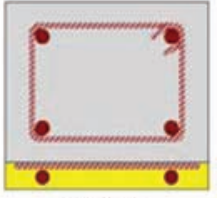

$2 \emptyset 10$

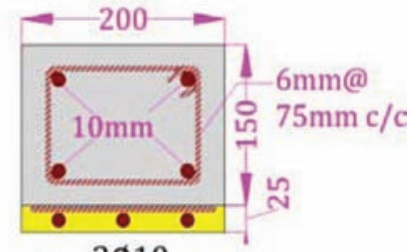

$3 \emptyset 10$

Cross section of all strengthened beams

Figure 15. Specimens strengthened by PCM overlaying. 
reinforcement amounts $(2 \phi 6,3 \phi 6,4 \phi 6)$, debonding in the case of $2 \phi 10$, and shear failure for the case with largest tension reinforcement amounts $(3 \phi 10)$ (see Figure 17). In the case of $2 \phi 10$, the failure mode was flexure-shear failure at $20^{\circ} \mathrm{C}$ but debonding at 40 and $60^{\circ} \mathrm{C}$, indicating that the debonding strength decreased with temperature increase. The model to predict the ultimate loads with flexure, shear, and debonding failure was proposed based on truss analogy where the proposed shear bond strength model was implemented (Figure 18). The prediction model can predict well not only the peak load but also failure mode for both cases with and without primer treatment and for all the temperature cases as shown in Figure 19.

\section{CONCLUDING REMARKS}

Based on the experimental investigations presented in this paper, the following conclusions can be drawn:

(1) Shear bond strength of FRP-concrete interface is affected by moisture because resin-concrete adhesion strength is affected by moisture. Among tested CFRP external bonding systems, wet-layup CFRP systems all show the strength reduction, while prefabricated CFRP systems all show the strength increase after immersion. The bond stress-slip relationship and interfacial fracture energy also change, which can explain the change in shear bond strength. The
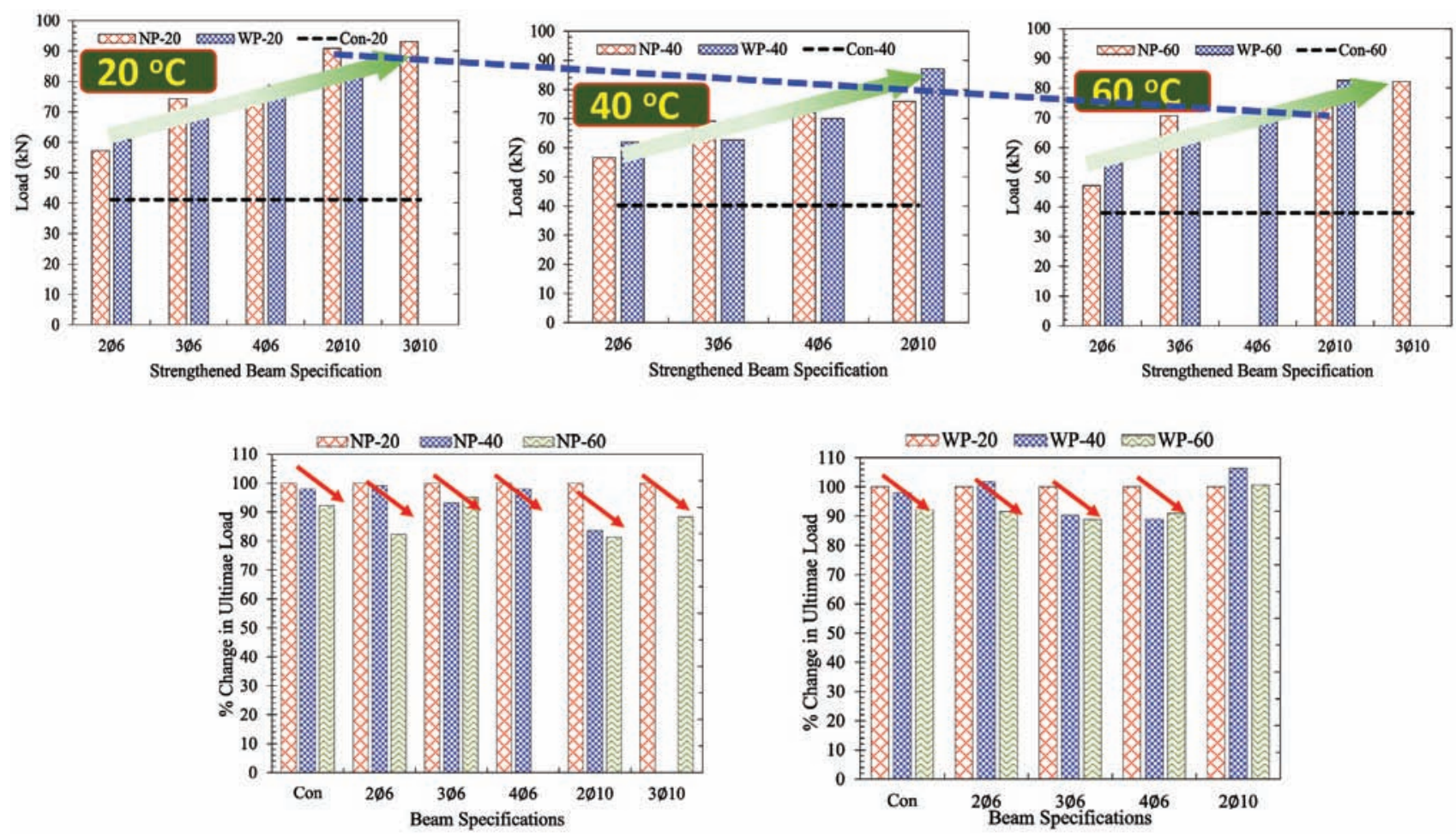

Figure 16. Effects of temperature and reinforcement amount in overlay on ultimate loads.

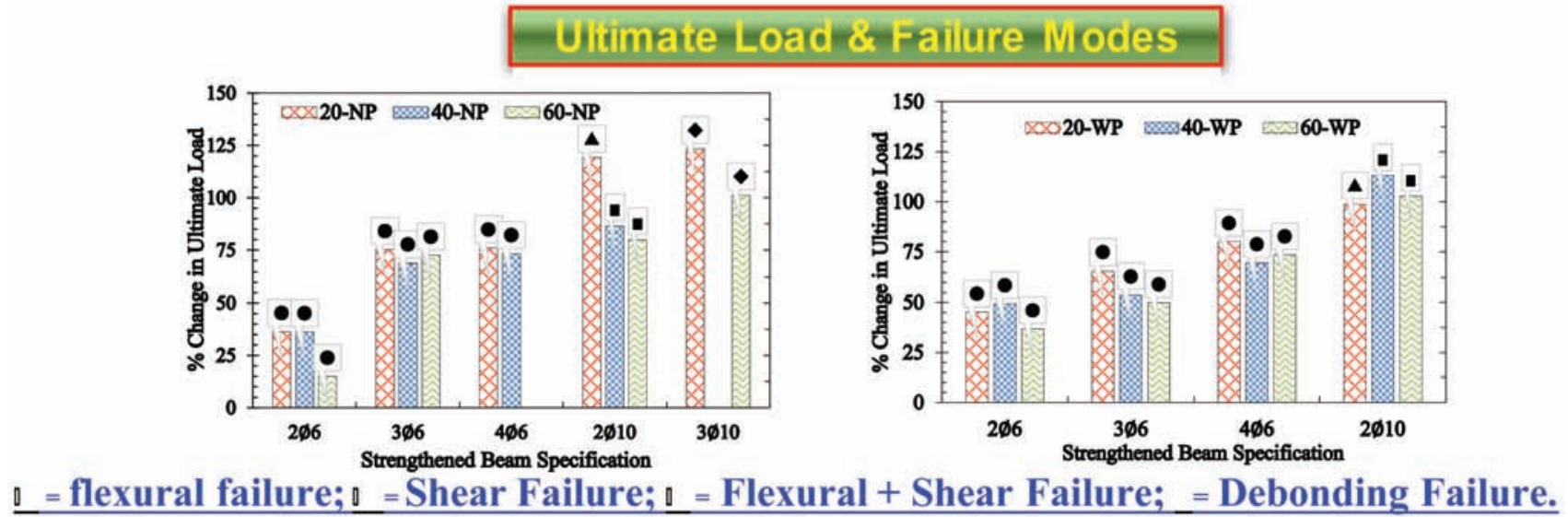

Figure 17. Ultimate loads and failure modes. 


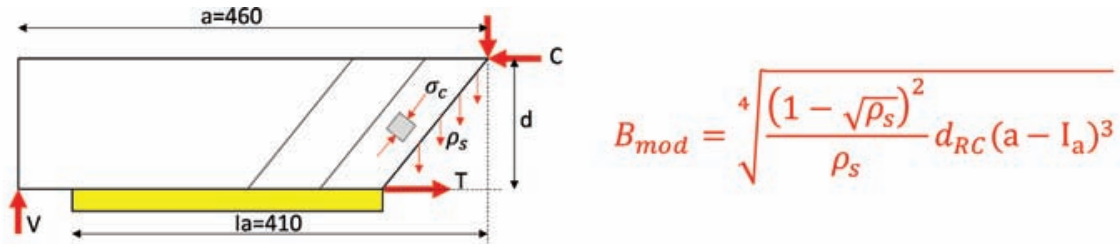

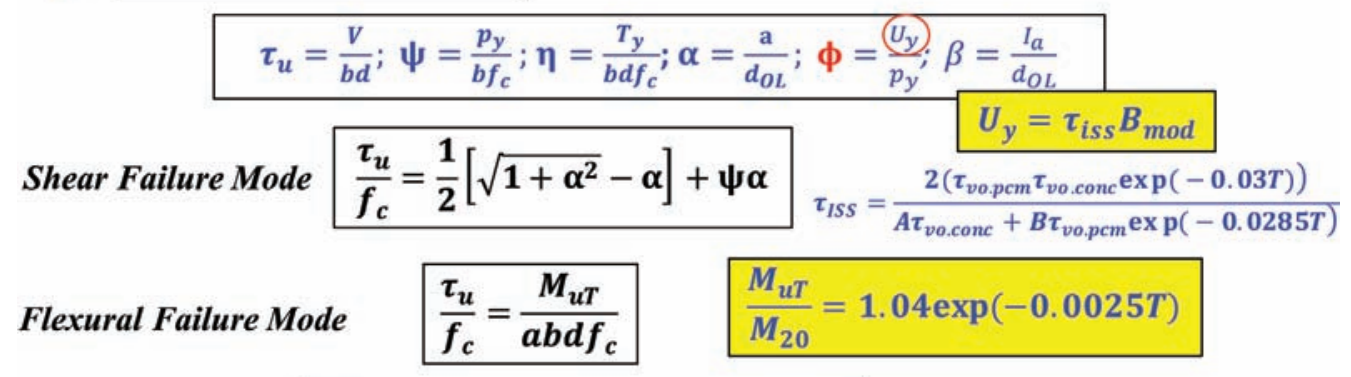

Debonding failure $\frac{\tau_{u}}{f_{c}}=\psi\left[\phi+\alpha-\sqrt{(\phi+\alpha)^{2}-2 \phi \beta}\right.$

Figure 18. Ultimate load prediction model based on truss analogy and shear bond strength.
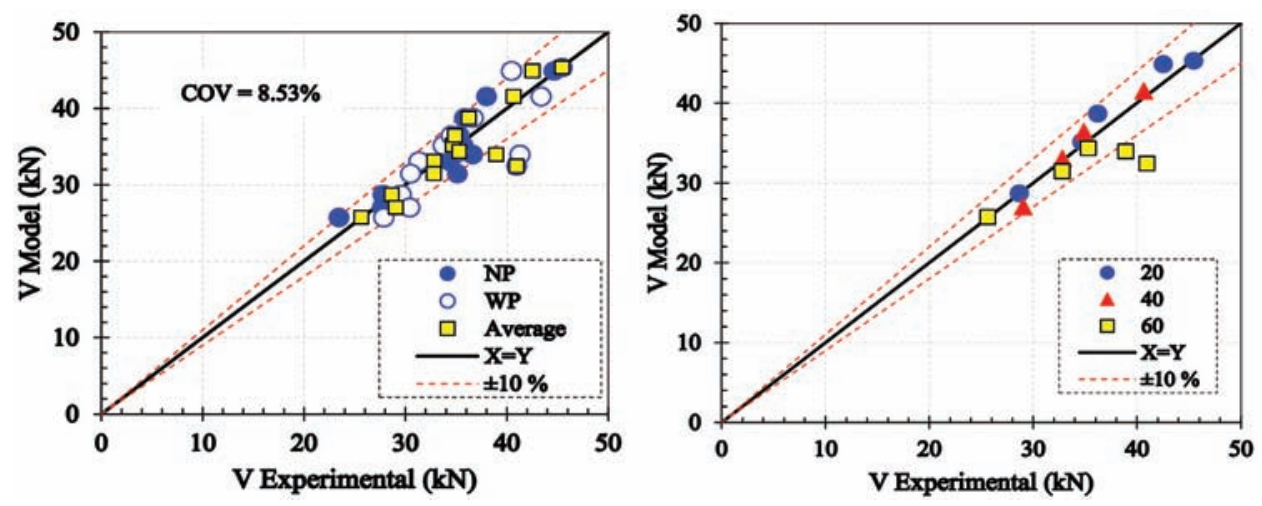

Figure 19. Comparison between predicted and experimental ultimate loads.

reduction in shear bond strength does not show clear dependency on resin strength/stiffness reduction. The observed big variation in shear bond strengths reported in past studies can be explained by difference in interface roughness, since chemical bond is affected by moisture but mechanical bond is not.

(2) Tension/shear bond strength of PCM-concrete interface is affected significantly by temperature but less by moisture. The tensile/shear bond strength is less than tensile/shear strength of constituent materials (PCM and concrete). The reduction in tension/shear bond strength depends on the reduction in tensile/shear strengths of PCM and concrete and then estimated by proposed equation which is a function of PCM and concrete strength. The ultimate loads of beams strengthened by PCM overlay can be predicted by the proposed model in which the proposed tensile/shear bond strength is applied.
Under high temperature, the failure mode can be changed from flexure/shear failure to debonding failure due to the reduction in shear bond strength.

\section{ACKNOWLEDGMENTS}

The authors are grateful to the Grant-in-Aid for Scientific Research (A) of Japan Society of Promotion of Science (No. 26249064), the Natural Science Foundation of China (Grant Nos. 51308494) and NEXCO Group Companies' Support Fund to Disaster Prevention Measures on Expressways as the financial supports to the studies in this paper.

\section{REFERENCE}

Dai, J. D., Ueda, T., \& Sato, Y. (2005). Development of the nonlinear bond stress-slip model of fiber reinforced plastics sheet-concrete interfaces with a simple method. Journal of Composites for Construction, 9(1), 52-62. 